\title{
PACKING AND COVERING BY TRANSLATES OF CERTAIN NONCONVEX BODIES
}

\author{
HUGH EVERETT AND DEAN HICKERSON
}

\begin{abstract}
We develop techniques for determining the packing and covering constants for star bodies composed of cubes.

In the theory of convex sets problems of tiling, packing, and covering by translates of a given set have a long history, with the main focus on the packing and covering by spheres. Only in a few cases is the densest packing or sparsest covering known, even in the case of the sphere, except, of course, when the set happens to tile Euclidean space. In a series of papers $\mathbf{S}$. $K$. Stein [4], [5], [6], [7] and W. Hamaker [1] used algebraic techniques in the problem of tiling Euclidean space of arbitrary dimension by translates of certain star bodies composed of cubes. The present paper has two purposes. First, it establishes a "Shift Theorem" that reduces tiling, packing, and covering problems for translates of a union of cubes to translates by vectors with integer coordinates. In a sense, this reduces continuous geometric problems in Euclidean space to discrete algebraic problems in a power of the infinite cyclic group. This theorem automatically generalizes many of Stein's results that depend on the assumption of integer translates. Second, it illustrates the Shift Theorem and a general "contribution" argument by determining the packing and covering constants for a particular star body.
\end{abstract}

1. Definitions. Consider in $n$-dimensional Euclidean space $R^{n}$ the set of closed unit cubes parallel to the coordinate axes and whose centers have integer coordinates. The union of a finite number of these cubes, one of which has its center at the origin, is called a cluster, and denoted $K$. A family of translates of $K$ whose union is $R^{n}$ is a covering of $R^{n}$. A family of translates of $K$ whose interiors are disjoint is a packing of $R^{n}$. A covering that is also a packing is a tiling. Packing density and covering density of $K$ are defined as they are for a convex body [3]. For $S \subseteq R^{n}$ the interior of $S$ will be denoted $\operatorname{int}(S)$.

2. The Shift Theorem. Let $A$ be a subset of $R^{n}$ homeomorphic to the ball $E^{n}$. Let $A$ lattice-tile $R^{n}$, that is, assume there is a lattice $L$ such that the family $\{l+A: l \in L\}$ tiles $R^{n}$. (For convenience, one may picture $A$ as a unit square in the plane and the lattice $L$ as the set of vectors with integer coordinates.) Let $K$ be the union of some of the sets $l+A$; that is, let $K=M+A$ for some $M \subseteq L$. We will show that the packing, covering, and tiling problems for $K$ can be reduced to the case where the translation vectors are elements of $L$.

Received by the editors August 10, 1978.

AMS (MOS) subject classifications (1970). Primary 52A45, $10 \mathrm{E} 30$.

Key words and phrases. Tiling, packing, covering, star body. 
Let $\mathscr{F}=\{w+K: w \in W\}$ be a denumerable family of translates of $K$. Since both $W$ and $L$ are denumerable, it is possible to translate the family $\mathscr{F}$ by some vector $c$ so that no $c+w$ with $w \in W$ is on the boundary of any $l+(-A), l \in L$. Let us assume this initial shift has been made, if necessary. Thus, we now assume that no $w \in W$ is on the boundary of any $l+(-A)$, $l \in L$.

Define the "shift" operation as follows. Consider the typical translate $w+K \in \mathscr{F}$. Since $\{l+(-A): l \in L\}$ is a tiling of $R^{n}$ and $w$ is not on the boundary of any $l+(-A)$, we must have $w \in \operatorname{int}(v+(-A))$ for a unique $v \in L$. Call $v$ the "shift of $w^{\prime}$ ". Let $\mathscr{F}^{\prime}=\{v+K: v$ is the shift of some $w \in W\}$. Note that $\mathscr{F}^{\prime}$ has the same upper and lower densities as $\mathscr{F}$.

THEOREM 2.1.

(1) If $\mathscr{F}$ is a packing, then $\mathscr{F}^{\prime}$ is a packing.

(2) If $\mathscr{F}$ is a covering, then $\mathscr{F}^{\prime}$ is a covering.

(3) If $\mathscr{F}$ is a tiling, then $\mathscr{F}^{\prime}$ is a tiling.

Proof. Note that (3) follows immediately from (1) and (2).

To prove (1), assume that $\mathscr{F}$ is a packing. We must show that if the interiors of $v_{1}+K$ and $v_{2}+K$ intersect, with $v_{i}+K \in \mathscr{F}^{\prime}, i=1$, 2, then $v_{1}=v_{2}$. So suppose that $v_{1}+k_{1}=v_{2}+k_{2}$ with $k_{i} \in \operatorname{int}(K)$.

We may assume that neither $k_{1}$ nor $k_{2}$ is on the boundary of any set $l+A$, $l \in L$. Since $K=M+A$, we have $k_{i}=m_{i}+a_{i}$, with $m_{i} \in M$ and $a_{i} \in$ $\operatorname{int}(A)$. Hence $\left(v_{1}+m_{1}\right)+a_{1}=\left(v_{2}+m_{2}\right)+a_{2}$. Since $v_{i}+m_{i} \in L$ and $a_{i} \in$ $\operatorname{int}(A)$, we must have $v_{1}+m_{1}=v_{2}+m_{2}$ and $a_{1}=a_{2}$.

Since $v_{i}+K \in \mathscr{F}^{\prime}, v_{i}$ must be the shift of some $w_{i} \in W$. Hence $w_{i} \in \operatorname{int}\left(v_{i}\right.$ $+(-A))$ so $w_{i}=v_{i}-b_{i}$ for some $b_{i} \in \operatorname{int}(A)$. Then

$$
w_{1}+\left(m_{1}+b_{1}\right)=v_{1}+m_{1}=v_{2}+m_{2}=w_{2}+\left(m_{2}+b_{2}\right) \text {. }
$$

Since $w_{i} \in W$ and $m_{i}+b_{i} \in \operatorname{int}(K)$ we have $w_{1}=w_{2}$ and $m_{1}+b_{1}=m_{2}+$ $b_{2}$. Since $m_{i} \in L$ and $b_{i} \in \operatorname{int}(A)$, we therefore have $m_{1}=m_{2}$ and $b_{1}=b_{2}$. But $v_{1}+m_{1}=v_{2}+m_{2}$ so $v_{1}=v_{2}$, as required.

To prove (2), let $p \in R^{n}$. We must show that $p \in v+K$, where $v$ is the shift of some $w \in W$. Let $p=l+a$ with $l \in L, a \in A$. Let $l=w+k$ with $w \in W, k \in K$. Let $k=m+b$ with $m \in M, b \in A$. Then $w=(l-m)-b$. Note that $b \in \operatorname{int}(A)$, since otherwise $w$ would be on the boundary of $(l-m)+(-A)$. Therefore, $l-m$ is the shift of $w$. But

$$
p=l+a=(l-m)+(m+a) \in(l-m)+K,
$$

so we are done.

In case $A$ is the unit cube, the Shift Theorem reduces the tiling, packing, or covering problem for a cluster to the corresponding problem for translates by vectors with integer entries. However, the shift of a lattice tiling by a cluster need not be a lattice tiling. For instance, the cluster in $R^{2}$ formed of two unit squares with centers at $(0,0)$ and $(2,0)$, tiles $R^{2}$ in a lattice manner (with the 
lattice generated by $(1,1 / 2)$ and $(4,0))$. On the other hand, no lattice of integer translates of this cluster tiles $R^{2}$.

Henceforth we will consider the translating vectors to have integer coordinates. Instead of packings or coverings of $R^{n}$, we consider analogously packings or coverings of $Z^{n}$, where $Z$ is the set of integers. Each point $P$ in $Z^{n}$ is thus identified with the unit cube parallel to the axes and having $P$ as center. Packing and covering constants are not changed by this restriction. However, lattice packing and lattice-covering constants may be changed.

3. Packing and covering by the $T$. To illustrate the Shift Theorem, we determine the packing and covering constants for the cluster in $R^{2}$ formed by the five unit squares whose centers are $(0,0),(0,1),(0,2),(1,2)$ and $(-1,2)$, called a $T$. It is easy to construct several lattice packings of $R^{2}$ by $T$ of density $5 / 6$. We show that any packing by $T$ has density at most $5 / 6$.

By the Shift Theorem, any packing of $R^{2}$ by translates of $T$ can be altered to a packing in which the translating vectors have integer coordinates, without changing the packing density. For any translate $T$ in this packing, there is an empty unit square just to the right of at least one of the two unit squares in the stem of the $T$. Hence the packing density is at most $5 / 6$.

It is easy to construct lattice coverings of $R^{2}$ by the $T$ of average density $5 / 4$. To show that any covering of $R^{2}$ by translates of $T$ has density at least $5 / 4$, we proceed in two steps. First, by the Shift Theorem, we consider only integer translates of the $T$. Second, we introduce the notion of "contributed square", which easily extends to coverings by integer translates of any cluster.

Consider a fixed covering family of distinct translates of $T$ by integer coordinates. Each such translate, $v+T$, contains five squares. A square $s$ in $v+T$ is contributed by $v+T$ if, of those translates that contain $s$, either

(a) $v+T$ is the unique one furthest right (i.e., $v$ has the largest $x$ coordinate),

or

(b) no translate of $T$ further right contains $s$, but other translates just as far right contain $s$, and $v+T$ is the lowest of those translates.

Note that $v+T$ can contribute anywhere from 0 to 5 squares.

Note also that each square is contributed by exactly one translate $v+T$.

Let $c$ be the average number of squares contributed per translate. (If this limit does not exist, let $c$ be the lim inf of the average number of squares contributed per tile.) We will show that $c \leqslant 4$. Thus the covering density is at least $5 / 4$.

To do this, we will show that for every $v+T$ that contributes five squares, there is associated in a well defined manner, a $w+T$ that contributes at most three squares.

For convenience, assume that $(0,0)+T$ contributes five squares. The square $s$ with center $(1,1)$ is covered by some translate $w+T$. But, since 
$(0,0)+T$ contributes five squares, $s$ must be covered by $(2,-1)+T$. Consider next the square $s^{*}$ with center $(1,0)$ and the translate $w^{*}+T$ that contributes $s^{*}$. By examining the five ways in which $s^{*}$ can be in $w^{*}+T$, and keeping in mind that $(0,0)+T$ contributes five squares, we see that $w^{*}+T$ must be $(2,-2)+T$. But this implies that $(2,-1)+T$ contributes at most three squares.

While the Shift Theorem was essential in determining the covering constant, it was merely a convenience in determining the packing constant. Without applying the Shift Theorem one could have shown that there is an uncovered set just to the right of the stem that has area at least one unit. (This uncovered set may not be connected.)

4. Packing and covering by crosses. Let $k$ be a nonnegative real number. A $k$-cross in the plane consists of a unit square together with four arms of length $k$ and width 1 . Its total area is therefore $4 k+1$. Note that for $k=1 / 2$ or 1 the $k$-cross tiles $R^{2}$.

THEOREM 4.1. For $k \geqslant 1$ the $k$-cross packs $R^{2}$ with density $(4 k+1) /\left(k^{2}+\right.$ $2 k+2)$. This is best possible.

Proof. A packing of the required density is provided by using as translating vectors those of the form $m(k+1,1)+n(-1, k+1)$, for integer $m$ and $n$.

To show that no packing can be denser, consider for any cross $c$ in the packing, the square of side $k$ situated just above the right arm of the cross and just to the right of the top arm of the cross. Disjoint translates of the $k$-cross, disjoint also from $c$, can cover an area of at most $2 k-1$ of this square of area $k^{2}$. Thus an area of at least $k^{2}-(2 k-1)$ is uncovered. From this, it follows that the density of any packing is at most $(4 k+1) /[4 k+1+$ $k^{2}-(2 k-1)$ ], from which Theorem 4.1 follows.

The covering constant for the $k$-cross is not determined in general. However, if $k$ is a positive integer, we conjecture that the covering constant is $(4 k+1) /(3 k+2)$. We construct a covering of that density, as follows.

For convenience, consider the $k$-cross with center $(0,0)$. Let $A$ be the union of the $k k$-crosses that have centers at $(0,0),(1,1),(2,2), \ldots$, and $(k-1, k$ -1 ). Note that $A$ tiles $R^{2}$ by translates. This tiling provides a covering of $R^{2}$ by translates of the $k$-cross.

A straightforward calculation shows that the area of $A$ is $k(3 k+2)$. Thus the density of the resulting covering of $R^{2}$ by $k$-crosses is

$$
\frac{k(4 k+1)}{k(3 k+2)}
$$

which is $(4 k+1) /(3 k+2)$. 
5. Problem. In addition to the various problems suggested by the earlier sections, we single out the following as perhaps most fundamental: Are the packing and covering constants of a cluster always rational numbers?

\section{REFERENCES}

1. W. Hamaker, Factoring groups and tiling space, Aequationes Math. 9 (1973), 145-149.

2. W. Hamaker and S. K. Stein, Splitting groups by integers, Proc. Amer. Math. Soc. 46 (1974), 322-324.

3. C. A. Rogers, Packing and covering, Cambridge Tracts in Math and Math. Physics, No. 54, Cambridge Univ. Press, New York, 1964.

4. S. K. Stein, Factoring by subsets, Pacific J. Math. 22 (1967), 523-541.

5. _ A symmetric star body that tiles but not as a lattice, Proc. Amer. Math. Soc. 36 (1972), 543-548.

6. , Tiling space by congruent polyhedra, Bull. Amer. Math. Soc. 80 (1974), 819-820.

7. __ Algebraic tiling, Amer. Math. Monthly 81 (1974), 445-462.

Department of Mathematics, University of California, Davis, California 95616 